\title{
Neutrophils exhibit distinct phenotypes toward chitosans with different degrees of deacetylation: implications for cartilage repair
}

\author{
Pascale Simard ${ }^{1,2 *}$, Hugo Galarneau1*, Sébastien Marois ${ }^{1}$, Daniel Rusu ${ }^{1,3}$, Caroline D Hoemann ${ }^{4}$, \\ Patrice E Poubelle ${ }^{1,3}$, Hani El-Gabalawy ${ }^{5}$ and Maria JG Fernandes ${ }^{1,2}$
}

\author{
${ }^{1}$ Centre de Recherche en Rhumatologie et Immunologie, Centre de Recherche du CHUQ-CHUL, boul. Laurier, Québec, G1V 4G2, Canada \\ 2Department of Anatomy and Physiology, Université Laval, avenue de la Médecine, Québec, G1V 0A6, Canada \\ ${ }^{3}$ Department of Medicine, Université Laval, avenue de la Médecine, Québec, G1V OA6, Canada \\ ${ }^{4}$ Department of Chemical Engineering, Institute of Biomedical Engineering, Ecole Polytechnique, boul. Édouard-Montpetit, Montréal, H3C 3A7, \\ Canada \\ ${ }^{5}$ Arthritis Centre, University of Manitoba, Sherbrook Street, Winnipeg, R3A 1M4, Canada \\ * Contributed equally
}

Corresponding author: Maria JG Fernandes, maria.fernandes@crchul.ulaval.ca

Received: 22 Sep 2008 Revisions requested: 17 Nov 2008 Revisions received: 23 Feb 2009 Accepted: 21 May 2009 Published: 21 May 2009

Arthritis Research \& Therapy 2009, 11:R74 (doi:10.1186/ar2703)

This article is online at: http://arthritis-research.com/content/11/3/R74

(c) 2009 Simard et al.; licensee BioMed Central Ltd.

This is an open access article distributed under the terms of the Creative Commons Attribution License (http://creativecommons.org/licenses/by/2.0), which permits unrestricted use, distribution, and reproduction in any medium, provided the original work is properly cited.

\begin{abstract}
Introduction Osteoarthritis is characterized by the progressive destruction of cartilage in the articular joints. Novel therapies that promote resurfacing of exposed bone in focal areas are of interest in osteoarthritis because they may delay the progression of this disabling disease in patients who develop focal lesions. Recently, the addition of $80 \%$ deacetylated chitosan to cartilage microfractures was shown to promote the regeneration of hyaline cartilage. The molecular mechanisms by which chitosan promotes cartilage regeneration remain unknown. Because neutrophils are transiently recruited to the microfracture site, the effect of $80 \%$ deacetylated chitosan on the function of neutrophils was investigated. Most studies on neutrophils use preparations of chitosan with an uncertain degree of deacetylation. For therapeutic purposes, it is of interest to determine whether the degree of deacetylation influences the response of neutrophils to chitosan. The effect of 95\% deacetylated chitosan on the function of neutrophils was therefore also investigated and compared with that of $80 \%$ deacetylated chitosan.
\end{abstract}

Methods Human blood neutrophils from healthy donors were isolated by centrifugation on Ficoll-Paque. Chemotaxis was performed using the chemoTX system. Production of superoxide anions was evaluated using the cytochrome $c$ reduction assay. Degranulation was determined by evaluating the release of myeloperoxidase and lactoferrin. The internalization of fluorescently labelled $80 \%$ deacetylated chitosan by neutrophils was studied by confocal microscopy.

Results Neutrophils were dose dependently attracted to $80 \%$ deacetylated chitosan. In contrast, 95\% deacetylated chitosan was not chemotactic for neutrophils. Moreover, the majority of the chemotactic effect of $80 \%$ deacetylated chitosan was mediated by phospholipase- $\mathrm{A}_{2}$-derived bioactive lipids. Contrary to the induction of chemotaxis, neither $80 \%$ nor $95 \%$ deacetylated chitosan activated the release of granule enzymes or the generation of active oxygen species. Despite the distinct response of neutrophils toward $80 \%$ and $95 \%$ deacetylated chitosan, both chitosans were internalized by neutrophils.

Conclusions Eighty per cent deacetylated chitosan induces a phenotype in neutrophils that is distinct from the classical phenotype induced by pro-inflammatory agents. Our observations also indicate that the degree of deacetylation is an important factor to consider in the use of chitosan as an accelerator of repair because neutrophils do not respond to 95\% deacetylated chitosan.

80 M: $80 \%$ deacetylated chitosan of medium viscosity; $95 \mathrm{M}: 95 \%$ deacetylated chitosan of medium viscosity; cPLA2- $\alpha$ : cytosolic phospholipase $\mathrm{A}_{2}-\alpha$; DDA: degree of deacetylation; FBS: fetal bovine serum; fMLP: $N$-formyl-methionyl-leucyl-phenylalanine; HTAB: hexadecyltrimethylammonium bromide; $\mathrm{LTB}_{4}$ : leukotriene $\mathrm{B}_{4}$; MPO: myeloperoxidase; OA: osteoarthritis; PAF: platelet-activating factor; PDI: polydispersity index; PMN: polymorphonuclear neutrophil; RITC: rhodamine B isothiocyanate. 


\section{Introduction}

Osteoarthritis (OA) is characterized by progressive destruction of cartilage in the articular joints [1]. Because it is one of the main causes of disability, this form of arthritis is a burden to both society and the patient. The incidence of OA increases with age. Over $80 \%$ of the elderly population exhibits radiographic evidence of $\mathrm{OA}$.

Focal cartilage lesions in humans can be treated by microfracture. This resurfacing procedure, when successful, can re-stabilize the joint and slow the progression of OA. Chitosan was recently shown to promote the regeneration of articular cartilage through the application of an in situ solidifying chitosanglycerol phosphate/blood clot over lesions treated with microfracture $[2,3]$. Chitosan-glycerol phosphate/blood clots represent a novel articular cartilage repair approach, which has yielded promising results in the clinic [4].

Chitosan is a linear polymer of $\beta(1 \rightarrow 4)$-linked glucosamine and $N$-acetyl-D-glucosamine residues obtained by the $N$ deacetylation of chitin. Chitosan is biodegradable, non-toxic, and nonimmunogenic [5-7]. The degree of deacetylation (DDA) influences the physical properties of chitosan. As the degree of deacetylation increases, the degree of solubility of chitosan in different solvents decreases and susceptibility to lysosomal biodegradation decreases $[8,9]$. The chitosan used in the cartilage repair model is of medium viscosity and is $80 \%$ deacetylated $(80 \mathrm{M})$. In vivo, $50 \%$ to $80 \%$ deacetylated chitosan is slowly degraded and eventually cleared by enzymatic and cell-based mechanisms $[5,10]$.

The presence of $80 \mathrm{M}$ chitosan over repairing microfracture or microdrill holes is associated with the recruitment of polymorphonuclear neutrophils (PMNs) to the granulation tissue as well as remodeling and revascularization of the damaged trabecular bone, and subsequent formation of more hyaline repair tissue in both rabbit and sheep repair models $[2,3,11]$. In contrast, few PMNs home to microdrills in the absence of chitosan [11]. Remarkably, PMNs persist in repairing defects for several weeks, in parallel with clearance of the $80 \mathrm{M}$ chitosan particles. Therefore, in contrast to traditional notions that persistence of PMNs in repairing wounds is detrimental to repair, in this cartilage repair model the persistence of PMNs during the first few weeks of repair is related to a more favorable cartilage repair outcome.

To identify the mechanisms through which $80 \mathrm{M}$ chitosan promotes cartilage regeneration in this repair model, the first objective of the present study was to investigate the effect of $80 \mathrm{M}$ chitosan on the function of PMNs. Even though the response of PMNs toward chitosan has been characterized in vitro to some extent [12-16], it remains difficult to compare the results between studies and to draw clear conclusions because the chitosan preparations in most studies vary and details on the quality of the chitosan preparations are rarely provided. With regard to the latter, the presence of endotoxins is an important consideration when investigating PMN responses. In the present study, chitosan preparations of medical grade were used. Regarding the former, some studies use chitosan preparations of unspecified DDA whereas other studies use water-soluble chitosan, which does not form a solid implant [16] or semi-crystalline scaffolds [10]. This complicates the interpretation of the results because the degree of DDA is a determining factor for the physical properties of chitosan, and it is not yet established to what extent PMNs respond differently to chitosans of different DDA. Also, it remains to be determined whether the PMN response varies toward chitosan presented as a particulate or a cross-linked scaffold. To optimize the use of chitosan in clinical applications, it is therefore critical to address the effect of DDA on the ability of chitosan to activate PMNs and to compare different preparations of chitosan (for instance, chitosan suspensions versus scaffolds). The second objective of this study was therefore to compare the response of PMNs to two chitosan preparations of a defined DDA, namely $80 \mathrm{M}$ chitosan and 95\% deacetylated (95 M) chitosan. The $95 \mathrm{M}$ chitosan was investigated because our preliminary results indicate that PMNs respond differently to chitosan of this DDA in vivo.

\section{Materials and methods}

All preparation and incubation procedures were performed under sterile conditions.

\section{Materials}

The two medical grade chitosan preparations $(80.6 \%$ or $94.6 \%$ DDA) used in this study are certified to contain under $0.2 \%$ weight/weight protein, $<500 \mathrm{EU} / \mathrm{g}$ endotoxin, and $<10$ parts/million heavy metals. To prevent contamination by endotoxin of chitosan solutions, chitosan powder was dissolved in double-distilled water filtered by MilliQ (Millipore, Billerica, MA, USA), at a resistance below $18.2 \mathrm{M} \Omega \mathrm{cm}$ and levels of trace organic compounds below 30 parts/billion with certified $1.0 \mathrm{~N}$ $\mathrm{HCl}$, using heat-treated endotoxin-free glassware and stir bars. Chitosan solutions were manipulated under aseptic conditions with laminar flow hoods and dispensed in sterile cryovials with cryo-resistance silicone gaskets for storage at $80^{\circ} \mathrm{C}$. The chitosan solutions are of medium viscosity of 1,422 $\mathrm{mPa}$.S for $80 \%$ DDA chitosan, termed $80 \mathrm{M}(M n=176 \mathrm{kDa}$, polydispersity index $[\mathrm{PDI}]=1.4)$, or $2,964 \mathrm{mPa}$.S for $95 \%$ DDA chitosan, termed $95 \mathrm{M}(M n=147 \mathrm{kDa}, \mathrm{PDI}=1.6)$, as previously described by $\mathrm{Ma}$ and coworkers [17]. Chitosan solutions were further diluted with sterile double-distilled water to $5 \mathrm{mg} / \mathrm{ml}$ or $0.5 \mathrm{mg} / \mathrm{ml}$ stock solutions. The DDA of chitosan was provided by the certificates of analysis from the supplier (Bio Syntech Canada, Inc., Laval, QC, Canada).

Rhodamine B isothiocyanate (RITC) was covalently conjugated with each chitosan (RITC-chitosan) to generate either RITC-80 M (80\% DDA, number average molecular weight $\mathrm{Mn}$ $=144 \mathrm{kDa}, \mathrm{PDI}=1.3,0.5 \% \mathrm{~mol} / \mathrm{mol} \mathrm{RITC} /$ chitosan) or RITC- 
$95 \mathrm{M}(95 \% \mathrm{DDA}, \mathrm{Mn}=177 \mathrm{kDa}, \mathrm{PDI}=1.1,0.6 \% \mathrm{~mol} / \mathrm{mol}$ RITC/chitosan). The DDA of RITC-chitosan derivatives is reported as unchanged, given that the derivatization level was determined as only $0.5 \% \mathrm{~mol}$ RITC/mol chitosan (as reported by Ma and coworkers [17]). Ficoll-Paque and dextran used for the isolation of PMN were obtained from Pharmacia (Kirkland, Québec, Canada) and fetal bovine serum (FBS) as well as RPMI 1640 were purchased from Wisent (St-Bruno, Québec, Canada). Calcein/AM was obtained from Invitrogen (Burlington, Ontario, Canada). Migration was assessed using the ChemoTx system from Neuroprobe (Gaithersburg, MD, USA) and the purified myeloperoxidase (MPO), O-dianisidine dihydrochloride, hydrogen peroxide, and cytochalasin B used in the MPO assay were obtained from Sigma-Aldrich (Oakville, Ontario, Canada). Hexadecyltrimethylammonium bromide (HTAB) was purchased from Fluka Chemie $\mathrm{GmbH}$ (Buchs, Switzerland). Cytochrome $c$ equine heart and pyrrolidine-1 were purchased from Calbiochem (Gibbstown, New Jersey, USA).

\section{Isolation of polymophonuclear neutrophils}

The Institutional Review Board of the Universite Laval (Québec, OC, Canada) approved the study, and volunteers signed a consent form. PMNs were isolated as previously described [18]. Briefly, venous blood was obtained from healthy adult volunteers, in accordance with instituteapproved protocols, in tubes containing heparin or isocitrate. No difference was observed between the results obtained with these two anticoagulants. After sedimentation of red blood cells in $2 \%$ dextran, PMNs were aseptically purified by centrifugation on Ficoll-Paque cushions. Contaminating erythrocytes were removed by hypotonic lysis and PMNs were resuspended in RPMI 1640 supplemented with 0.1\% FBS previously decomplemented at $56^{\circ} \mathrm{C}$ for 30 minutes, except for the chemotaxis experiment (10\% decomplemented FBS).

\section{Chemotaxis}

Chemotaxis was measured as described previously [13]. Briefly, PMNs were resuspended in RPMI-1640 and 10\% FBS at a concentration of $10^{7} \mathrm{cells} / \mathrm{ml}$ and were pre-incubated with $1 \mu \mathrm{g} / \mathrm{ml}$ calcein/AM at $37^{\circ} \mathrm{C}$ for 30 minutes in the dark with constant agitation. Cells were washed twice and resuspended in RPMl $/ 10 \% \mathrm{FBS}$ at $5 \times 10^{7} \mathrm{cell} / \mathrm{s} / \mathrm{ml}$ at $37^{\circ} \mathrm{C}$. PMN migration was monitored using a 96-well ChemoTx disposable chemotaxis system. The fluorescence of cells in the filters was measured using a microplate fluorescence reader (FL600; Bio-Tek Instruments, Winooski, VT, USA; excitation wavelength 485 $\mathrm{nm}$, emission wavelength $530 \mathrm{~nm}$ ). Fluorescence was converted to numbers of PMNs based on a standard curve generated by seeding known numbers of PMNs in the bottom of the chamber. The results are expressed as percentage of migrated cells, calculated as the fluorescence of migrated $\mathrm{PMNs} /$ fluorescence of $20,000 \mathrm{PMNs} / \mathrm{ml} \times 100$, obtained from the standard curve. In some experiments, PMNs were incubated with a final concentration of $0.5 \mu \mathrm{g} / \mathrm{ml}$ pertussis toxin for
90 minutes or with $10^{-7} \mathrm{~mol} / /$ pyrrolidine -1 for 10 minutes at $37^{\circ} \mathrm{C}$ before the chemotaxis assay.

\section{Production of superoxide anions by polymorphonuclear neutrophils}

Superoxide anion production in response to $80 \mathrm{M}$ and $95 \mathrm{M}$ chitosan was determined using the cytochrome $c$ reduction assay, as previously described [19]. Briefly, freshly isolated PMNs were resuspended at a concentration of $1 \times 10^{6}$ cells/ $\mathrm{ml}$ in RPMI supplemented with $0.1 \%$ decomplemented FBS and cytochrome $c$ at a final concentration of $125 \mu \mathrm{g} / \mathrm{ml}$. The cells were incubated at $37^{\circ} \mathrm{C}$ for 5 minutes before the addition of the indicated concentrations of $80 \mathrm{M}$ or $95 \mathrm{M}$ chitosan. Cells were incubated for an additional 10 minutes at $37^{\circ} \mathrm{C}$ and the reaction stopped on ice for 10 minutes. The samples were then centrifuged at $12,000 \mathrm{~g}$ for 2 minutes at $4^{\circ} \mathrm{C}$ and the optical density of the supernatants was read at 540,550 , and 560 $\mathrm{nm}$ in a spectrophotometer (Milton Roy Spectronic 1001 Plus spectrophotometer, Milton Roy, Rochester, NY, USA). The amount of superoxide produced was calculated using the following formula: $A_{550}-\left(\left[A_{540}+A_{560}\right] / 2\right)$. The absorbance was transformed into the amount of superoxide produced $(\mathrm{nmol} /$ $10^{6}$ neutrophils) by using a conversion factor of 47.4 , derived from the molar extinction coefficient of cytochrome $c$.

\section{Release of myeloperoxidase and lactoferrin by polymorphonuclear neutrophils}

Degranulation was determined using the MPO and lactoferrin assay, as described by Bradley and coworkers [20] and Mocsai and colleagues [21], respectively.

For the MPO assay, PMNs $\left(10^{7}\right.$ cells $\left./ \mathrm{ml}\right)$ were incubated with $10 \mu \mathrm{mol} / \mathrm{l}$ cytochalasin $\mathrm{B}$, an actin depolymerizing agent that is known to amplify granule exocytosis, for 2 minutes at $37^{\circ} \mathrm{C}$ and then with the indicated concentrations of $80 \mathrm{M}$ or $95 \mathrm{M}$ chitosan for 30 minutes at $37^{\circ} \mathrm{C}$. A negative control with cytochalasin $\mathrm{B}$ and a positive control with cytochalasin $\mathrm{B}+\mathrm{N}$ formyl-methionyl-leucyl-phenylalanine (fMLP; 5 minutes incubation) were also performed. PMNs were then centrifuged for 1 minute at $12,000 \mathrm{~g}$ and lysed in HTAB lysis buffer $(0.5 \%$ $\mathrm{HTAB}, 50 \mathrm{mmol} / \mathrm{l} \mathrm{K}_{2} \mathrm{HPO}_{4}$ buffer $[\mathrm{pH} 6.0]$ ). One hundred microliters of the lysate and cell pellet was mixed with $2.4 \mathrm{ml}$ of a $\mathrm{K}_{2} \mathrm{HPO}_{4}$ buffer ( $50 \mathrm{mmol} / \mathrm{l}$ phosphate buffer [pH 6.0], 0.2 $\mathrm{mg} / \mathrm{ml}$ o-dianisidine dihydrochloride, and $0.003 \%$ hydrogen peroxide) before reading the optical density at $460 \mathrm{~nm}$ in a spectrophotometer (Milton Roy Spectronic 1001 Plus spectrophotometer). Purified human MPO (from 0.0625 to $1 \mathrm{U}$ ) was used to generate a standard curve. The value ' $\%$ release of MPO' corresponds to the ratio of the amount of MPO released/the total amount of released and cellular MPO.

For the lactoferrin assay, PMNs $\left(5 \times 10^{5}\right.$ cells $\left./ \mathrm{ml}\right)$ were incubated as described above for the MPO experiments. The release of lactoferrin was determined by enzyme-linked immunosorbent assay [21]. Supernatants were diluted 10-fold and 
100 -fold in $50 \mathrm{mmol} / / \mathrm{CO}_{2} / \mathrm{HCO}_{3}$ buffer $(\mathrm{pH}$ 9.6) and $100 \mu \mathrm{l}$ of the diluted supernatants or of known concentrations of human lactoferrin were added to 96-microwell plates (Nalge Nunc International, Rochester, NY, USA) and incubated overnight at $4^{\circ} \mathrm{C}$. Nonspecific binding sites were blocked with phosphate-buffered saline supplemented with $0.5 \%$ bovine serum albumin and $0.5 \%$ Tween- 20 overnight at room temperature. One hundred microliters of rabbit anti-human lactoferrin antibody (1:500 dilution of $17.05 \mathrm{mg} / \mathrm{ml}$ stock) was then added to each well and incubated for 2 hours. One hundred microliters of the secondary antibody, peroxidase-conjugated anti-rabbit antibody (1:40,000 dilution), was then added and incubated for 30 minutes. Each of the above steps was performed at room temperature and between each step the plates were repeatedly washed with $1 \times$ Tris-buffered saline/0.1\% Tween-20. Tetra-methyl-benzidene was added before stopping the reaction with $50 \mu \mathrm{l}$ of $1 \mathrm{~mol} / \mathrm{l}$ sulfuric acid. Absorbance was read at $450 \mathrm{~nm}$ with a microplate reader, and the lactoferrin concentration was calculated using the standard curve.

\section{Internalization of $80 \mathrm{M}$ and $95 \mathrm{M}$ chitosan by polymorphonuclear neutrophils}

Freshly isolated PMNs were resuspended in RPMI supplemented with $0.1 \%$ decomplemented autologous serum, prestained with $1 \mu \mathrm{g} / \mathrm{ml}$ calcein $/ \mathrm{AM}$ for 30 minutes at $37^{\circ} \mathrm{C}$, and then incubated with $30 \mu \mathrm{g} / \mathrm{ml}$ of RITC-80 M or RITC-95 M for 3 hours at $37^{\circ} \mathrm{C}$. PMNs were then centrifuged for 2 minutes at $1,500 \mathrm{~g}$ at room temperature and plated on a glass slide coated with 100\% decomplemented autologous serum. Slides were coated with autologous serum that was prepared by centrifuging clotted blood for 15 minutes at $700 \mathrm{~g}$ at room temperature and decomplemented for 30 minutes at $56^{\circ} \mathrm{C}$ to avoid activation of PMNs by the glass surface of the slide. PMNs were visualized live at $37^{\circ} \mathrm{C}$ in an environment chamber with $5 \% \mathrm{CO}_{2}$ through a spinning disc confocal microscope equipped with a $63 \times$ objective (Quorum Spinning Disc Wave FX, ON, Canada). The index of internalization of chitosan was calculated as the percentage age of PMNs that internalized any visually detectable quantity of RITC-chitosan. One hundred cells were observed for each experimental condition.

\section{Interaction of $80 \mathrm{M}$ and $95 \mathrm{M}$ chitosan with monocytes, granulocytes, and lymphocytes in whole blood}

The interaction of RITC-80 M and RITC-95 M chitosan with blood cells was determined by flow cytometry. Blood samples were first treated to eliminate erythrocytes by lysis, as described by Desmeules and coworkers [22], and were then stimulated for 30 minutes at $37^{\circ} \mathrm{C}$ with $5 \mu \mathrm{g} / \mathrm{ml} \mathrm{RITC}-80 \mathrm{M}$. Chitosan binding to cells was visualized using FACScan flow cytometry. The binding index was calculated as fluorescence units of a cellular population incubated with RITC-80 M chitosan/fluorescence units of a cellular population incubated in the same volume of the diluent (double-distilled water).

\section{Statistical analysis}

Results are expressed as means \pm standard error. Statistical analyses were performed using GraphPad Instat 3.0 (GraphPad Software, Inc., San Diego, CA, USA). Comparisons made between two groups were analyzed with the unpaired Student's $t$-test. Comparisons made between two or more groups were analyzed by one-way analysis of variance and the TukeyKramer post hoc test. $P<0.05$ was regarded to indicate statistical significance.

\section{Results}

The chemotactic effect of $80 \mathrm{M}$ and $95 \mathrm{M}$ chitosan on polymorphonuclear neutrophils

Before we conducted the study, we verified that we could reproduce the chemotactic activity of $80 \mathrm{M}$ chitosan toward PMNs observed in vivo, in the cartilage repair model, with an in vitro chemotaxis assay. Briefly, isolated PMNs were labeled with calcein/AM and the chemotactic activity of $80 \mathrm{M}$ chitosan was determined using the ChemoTx chemotaxis system - a transwell migration assay. We provide direct evidence that under our experimental conditions the $80 \mathrm{M}$ chitosan preparation was chemotactic for PMNs (Figure 1).

Chitosan preparations composed of chitosan of varying degrees of DDA, greater than $80 \%$, have been reported to be chemotactic for PMNs in vitro and in vivo [12]. It is not clear from these studies, however, whether the chemotactic activity of chitosan is dependent on the degree of DDA. To determine whether the chemotaxis of PMNs toward chitosan is dependent on the degree of DDA, a similar chemotaxis experiment was performed with $95 \mathrm{M}$ chitosan. In contrast to $80 \mathrm{M}$ chitosan, $95 \mathrm{M}$ chitosan was not chemotactic for PMNs under the same experimental conditions (Figure 1). These results not only confirmed the potential of $80 \mathrm{M}$ chitosan to attract PMNs but also indicated that the degree of acetylation of chitosan affected its chemotactic activity toward PMNs.

\section{Mediator(s) of the chemotactic effect of $80 \mathrm{M}$ and $95 \mathrm{M}$ chitosan on polymorphonuclear neutrophils}

We then investigated the molecular mechanism through which $80 \mathrm{M}$ chitosan induces chemotaxis of PMNs. Because neutrophils are a major source of the strong chemotactic mediators leukotriene $\mathrm{B}_{4}\left(\mathrm{LTB}_{4}\right)$ and platelet-activating factor (PAF), we studied the activation of this metabolic pathway in response to $80 \mathrm{M}$ chitosan. $\mathrm{LTB}_{4}$ is generated by the oxygenation of arachidonic acid by a 5-lipoxygenase. Arachidonic acid becomes available to 5-lipoxygenase once it is released from 1-O-alkyl-2-acyl-glycerophosphocholine by cytosolic phospholipase $\mathrm{A}_{2}-\alpha\left(\mathrm{cPLA}_{2}-\alpha\right)$ that also releases lyso-PAF simultaneously [23]. To determine whether these phospholipid-derived metabolites are responsible for the chemotactic activity of $80 \mathrm{M}$ chitosan toward human PMNs, the effect of pyrrolidine-1, an inhibitor of $\mathrm{CPLA}_{2}-\alpha$, on the chemotaxis of PMNs induced by $80 \mathrm{M}$ chitosan was determined. Briefly, PMNs were pre-incubated with pyrrolidine-1 and then allowed 
Figure 1
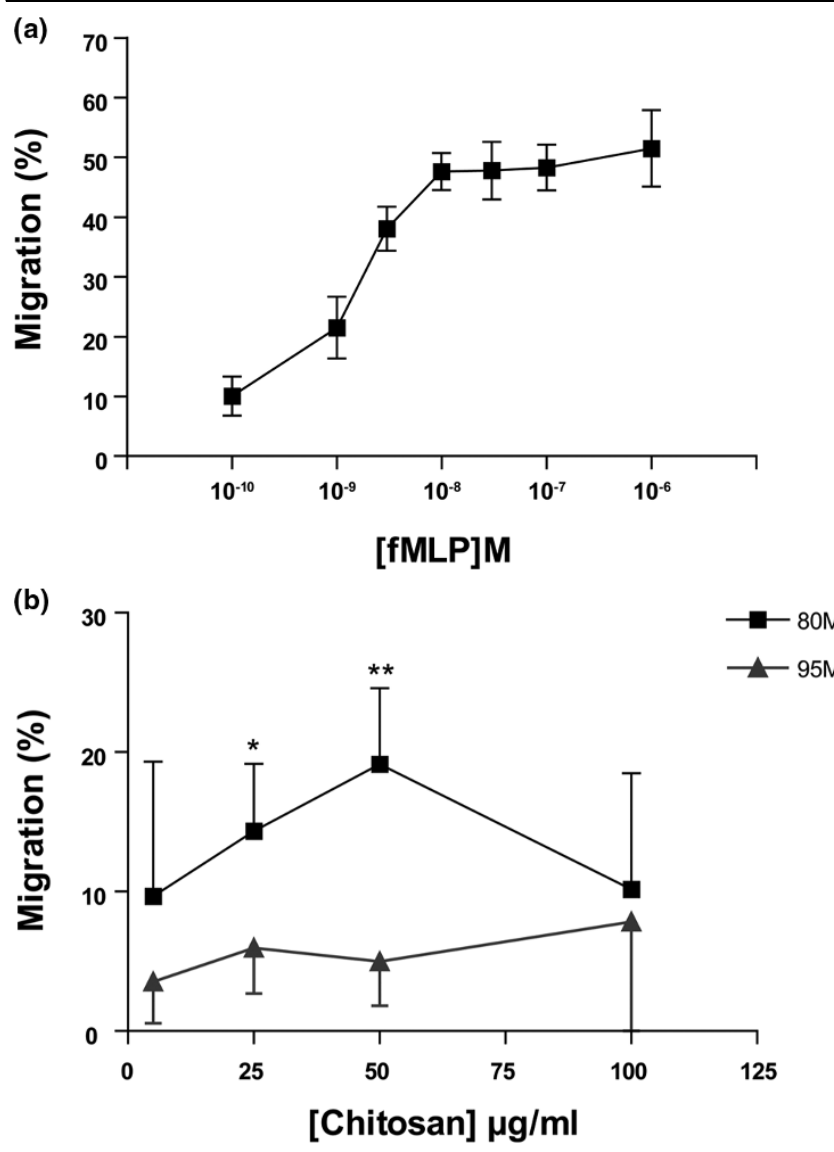

The chemotactic effect of chitosan on PMNs. Freshly isolated polymorphonuclear neutrophils (PMNs) were pre-stained with $1 \mu \mathrm{g} / \mathrm{ml}$ calcein$\mathrm{AM}$ and seeded on a polycarbonate filter placed above a well containing (a) $\mathrm{N}$-formyl-methionyl-leucyl-phenylalanine (fMLP; positive control) or (b) $80 \%$ deacetylated ( $80 \mathrm{M}$; black line) or $95 \%$ deacetylated (95 M; gray line) chitosan. PMNs were allowed to migrate for 1 hour before assessing migration, as described in 'Materials and Methods'. Percentage migration of PMNs $=$ the fluorescence of migrated PMNs/fluorescence of $20,000 \mathrm{PMNs} / \mathrm{ml} \times 100$, obtained from the standard curve. Results are presented as mean \pm standard error. $P$ values from Student's two-tailed unpaired $t$-test: ${ }^{\star} P=0.01$, fluorescence of PMNs migrated toward $25 \mu \mathrm{g} / \mathrm{ml} 80 \mathrm{M}$ chitosan versus fluorescence of PMNs migrated toward RPMI (Ctrl-); ${ }^{\star \star} P=0.004$, fluorescence of PMNs migrated toward $50 \mu \mathrm{g} / \mathrm{ml} 80 \mathrm{M}$ chitosan versus fluorescence of PMNs migrated toward RPMI. The positive control is the AMLP curve. This figure represents the results of three independent experiments.

to migrate toward $80 \mathrm{M}$ chitosan. Pyrrolidine-1 decreased the chemotaxis of PMNs toward $80 \mathrm{M}$ chitosan by $50 \%$ (Figure 2). These findings indicate that arachidonic acid metabolites are responsible, at least in part, for the chemotactic activity of 80 $M$ chitosan toward PMNs. As a general rule, $P M N$ chemotactic factors (for example, fMLP, interleukin-8, PAF, and $\mathrm{LTB}_{4}$ ) bind G-protein-coupled receptors. The activation of these G-protein-coupled receptors can be inhibited by pertussis toxin. We provide direct evidence that pertussis toxin significantly inhib-
Figure 2

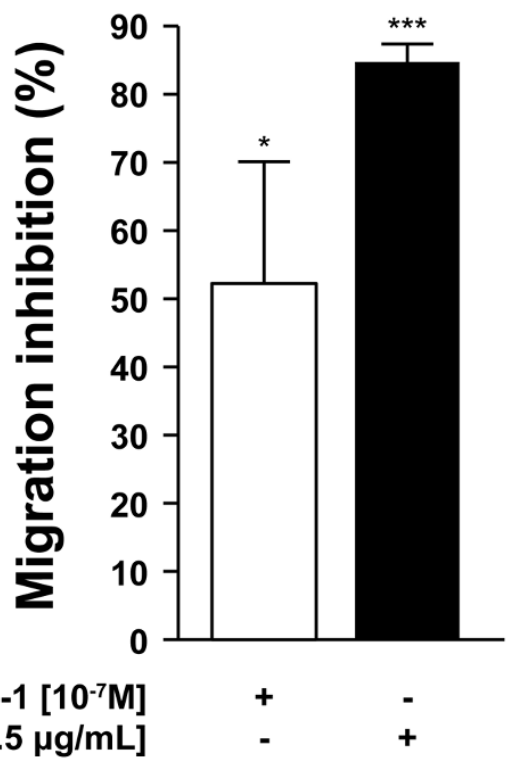

The effect of inhibitors on PMN chemotaxis towards chitosan. Freshly isolated polymorphonuclear neutrophils (PMNs) were resuspended in RPMI 1640 supplemented with $0.1 \%$ decomplemented fetal bovine serum, pre-stained with $1 \mu \mathrm{g} / \mathrm{ml}$ calcein-AM for 30 minutes at $37^{\circ} \mathrm{C}$ and incubated with $0.5 \mu \mathrm{g} / \mathrm{ml}$ pertussis toxin for 90 minutes and seeded on a polycarbonate filter above a well containing $50 \mu \mathrm{g} / \mathrm{ml} 80 \%$ deacetylated $(80 \mathrm{M})$ chitosan. Alternatively, PMNs were incubated with $10^{-7} \mathrm{~mol} / \mathrm{l}$ pyrrolidine for the last 10 minutes of the incubation with calcein-AM. Chemotaxis was performed as described in Figure 1. The percentage inhibition of migration corresponds to the fluorescence of PMNs incubated with the inhibitors that migrated toward $80 \mathrm{M}$ chitosan versus fluorescence of PMN incubated in media that migrated toward $80 \mathrm{M}$ chitosan. This figure represents the results of at least three independent experiments. ${ }^{\star} P=0.02$ and ${ }^{\star \star \star} P=0.0001$.

ited the chemotaxis of PMNs toward $80 \mathrm{M}$ chitosan by $80 \%$ (Figure 2).

\section{Production of superoxide anions and degranulation by polymorphonuclear neutrophils in response to $80 \mathrm{M}$ and $95 \mathrm{M}$ chitosan}

The mechanisms by which PMNs are thought to impair healing include the production of reactive oxygen species and the release of granule contents. Because PMNs promote wound healing and cartilage regeneration in the presence of $80 \mathrm{M}$ chitosan, it is of interest to determine whether - in the presence of $80 \mathrm{M}$ chitosan - PMNs produce these microbicidal substances. PMNs produce superoxide in response to $\mathrm{FMLP}$, a bacterial-derived antigen (Figure 3 ). In contrast to the large superoxide burst observed in response to $\mathrm{FLP}$, neither $80 \mathrm{M}$ (Figure 3a) nor $95 \mathrm{M}$ (Figure 3b) chitosan induced the release of superoxide by PMNs at all of the concentrations of chitosan tested (from 10 to $100 \mu \mathrm{g} / \mathrm{ml}$ ). The amounts of superoxide released by PMNs incubated with $80 \mathrm{M}$ or $95 \mathrm{M}$ chitosan were comparable to those of the negative control. 
Figure 3
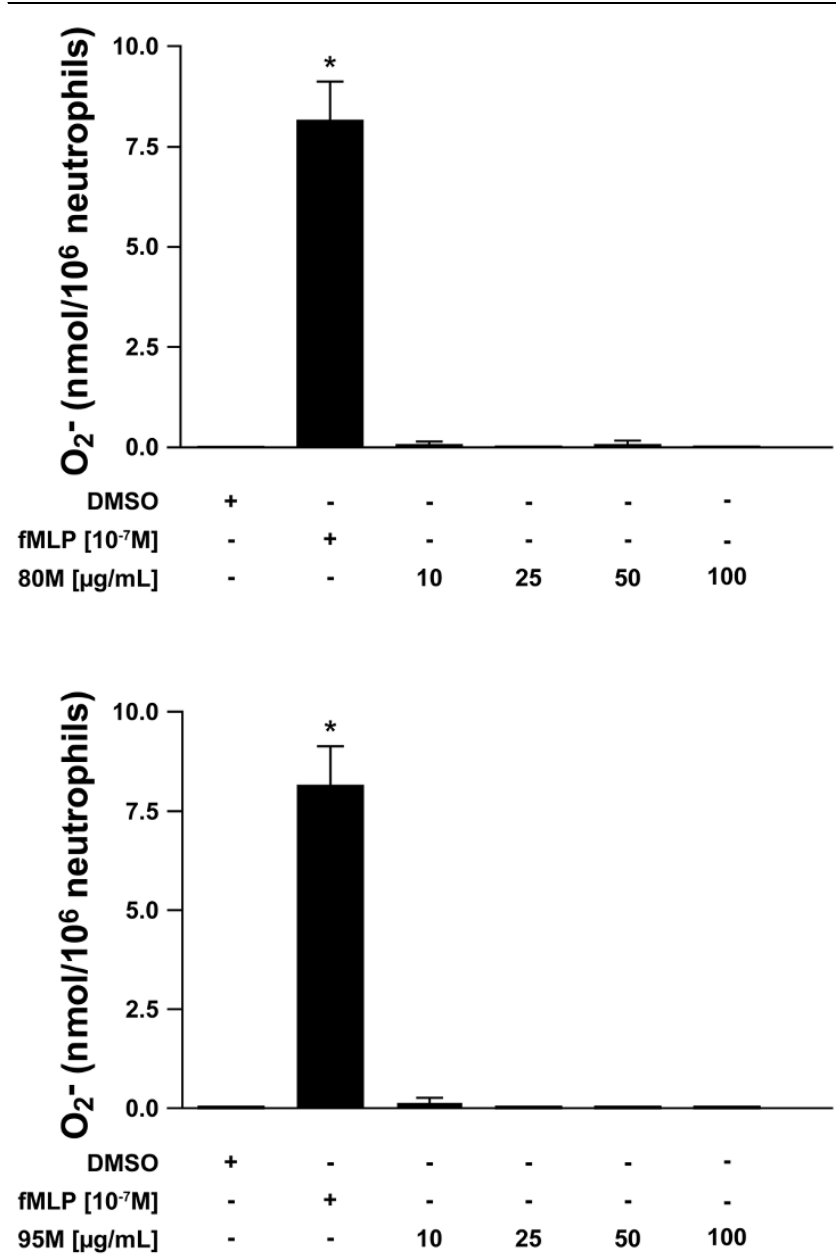

Production of superoxide anions by PMN in response to chitosan. Superoxide anion production was determined using the cytochrome $c$ reduction assay. Freshly isolated polymorphonuclear neutrophils (PMNs) resuspended in RPMI 1640 supplemented with $0.1 \%$ decomplemented fetal bovine serum were incubated with the indicated concentrations of $80 \%$ deacetylated $(80 \mathrm{M})$ or $95 \%$ deacetylated $(95 \mathrm{M})$ chitosan for 10 minutes at $37^{\circ} \mathrm{C}$. Results are presented as mean \pm standard error. The difference from the negative control is statistically significant: ${ }^{*} P<0.001$ (Tukey-Kramer test). The negative control $=$ PMNs incubated in Hanks' Balanced Salt Solution supplemented with $0.1 \%$ decomplemented fetal bovine serum and incubated with diluent (dimethyl sulfoxide [DMSO]). This figure represents the results of at least three independent experiments. fMLP, $N$-formyl-methionyl-leucylphenylalanine.

To determine whether $80 \mathrm{M}$ and $95 \mathrm{M}$ chitosan induce PMNs to degranulate, the release of the contents of the primary and secondary granules was determined by measuring the amount of MPO and lactoferrin released. When incubated with 10 to $100 \mu \mathrm{g} / \mathrm{ml} 80 \mathrm{M}$ or $95 \mathrm{M}$ chitosan, the amounts of MPO and lactoferrin released into the media by $\mathrm{PMNs}$ were negligible (Figure 4). Together, the above observations indicate that the effects of $80 \mathrm{M}$ chitosan on PMNs are not associated with the release of granule substances from $\mathrm{PMNs}$.

\section{The interaction of $80 \mathrm{M}$ and $95 \mathrm{M}$ chitosan with polymorphonuclear neutrophils}

The difference in chemotactic activity between $80 \mathrm{M}$ and 95 $\mathrm{M}$ chitosan toward PMNs may be due to the inability of PMNs to bind and/or internalize $95 \mathrm{M}$ chitosan. The binding and internalization of RITC-80 M and RITC-95 M chitosan by PMNs was investigated by live cell confocal microscopy. Live cell imaging revealed that PMNs internalized both RITC-80 M and RITC-95 $M$ chitosan in the presence of decomplemented serum, although internalization was much greater for fluorescent zymosan under similar conditions (Figure 5).

Because all of the white blood cells are present at the microfracture sites and could be involved in the effects of chitosan on cartilage repair and wound healing, we also investigated the ability of $80 \mathrm{M}$ chitosan to interact with other leukocytes. To observe the interaction of $80 \mathrm{M}$ chitosan with leukocytes with the same differential ratio in which these cells normally coexist, this analysis was performed in whole blood devoid of erythrocytes. Flow cytometry analysis of leukocytes in whole blood revealed that a greater amount of RITC-80 M chitosan associates with monocytes than granulocytes and lymphocytes (Figure 6a). Confocal microscopy revealed that monocytes readily internalize large amounts of RITC-80 M and RITC-95 M chitosan (Figure 6b).

\section{Discussion}

Novel therapeutic modalities that promote cartilage regeneration have the potential to delay significantly the progression of OA in patients who develop focal lesions. We therefore investigated some of the molecular mechanisms involved in the clinically beneficial effects of $80 \mathrm{M}$ chitosan, which was recently shown to promote cartilage regeneration in both large and small animal cartilage repair models $[2,3]$. In recent years evidence has accumulated that the PMN is more than just a leukocyte that phagocytoses foreign antigens. PMNs differentiate into dendritic cells [24] and have the capacity to modulate the adaptive immune response. The PMN therefore adopts different phenotypes that are determined by its environment. In this light, the response of PMNs to $80 \mathrm{M}$ chitosan was investigated to identify the characteristics of the phenotype of PMNs that promotes cartilage regeneration. We report that $80 \mathrm{M}$ chitosan selectively activates a subset of PMN functional responses.

The distinct phenotype of PMNs in response to $80 \mathrm{M}$ chitosan is characterized by PMN chemotaxis and the absence of the production of superoxide and degranulation. In comparison, $\mathrm{fMLP}$, a bacterial-derived peptide, is not only chemotactic for PMNs but also stimulates them to produce superoxide and degranulate. These observations strongly suggest that the PMN phenotype in the presence of $80 \mathrm{M}$ chitosan promotes repair due, at least in part, to the lack of superoxide production and PMN degranulation. Our data agree with a recent report indicating that water-soluble chitosan oligomers suppress the 

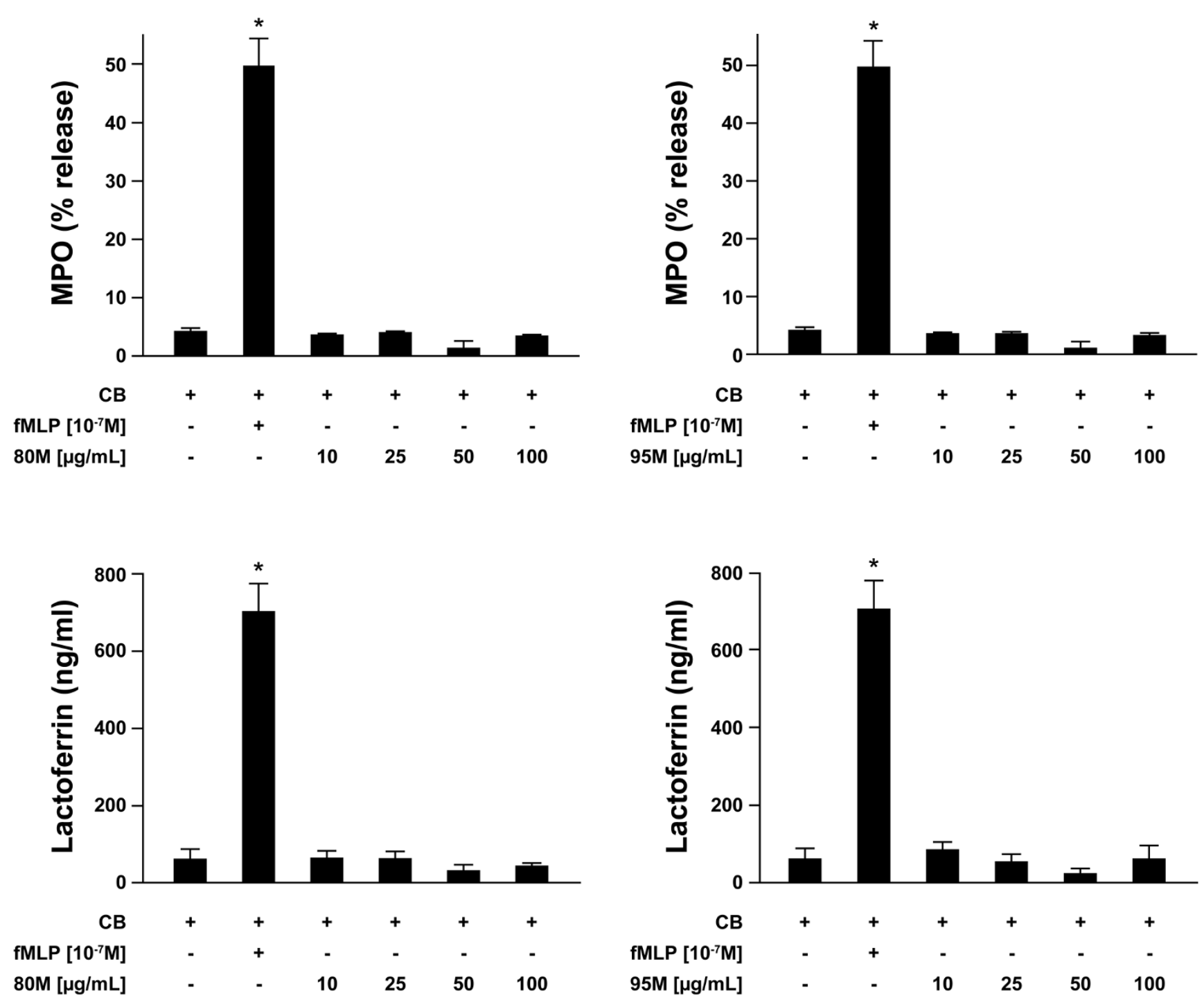

Release of myeloperoxidase and lactoferrin by PMNs in response of chitosan. Degranulation was determined using the (a, b) myeloperoxidase (MPO) and (c, d) lactoferrin assay, as described in 'Materials and Methods'. Freshly isolated polymorphonuclear neutrophils (PMNs) resuspended in RPMI 1640 supplemented with $0.1 \%$ decomplemented fetal bovine serum were treated with cytochalasin $B$ and further incubated with the indicated concentrations of $80 \%$ deacetylated $\left(80 \mathrm{M}\right.$; panels a and c) or $95 \%$ deacetylated $\left(95 \mathrm{M}\right.$; panels b and d) chitosan for 30 minutes at $37^{\circ} \mathrm{C}$. The quantity of MPO released is expressed as '\% MPO', which corresponds to the ratio of the amount of MPO released/total amount of cellular MPO. The amount of lactoferrin released is expressed in $\mathrm{ng} / \mathrm{ml}$. Results are presented as mean \pm standard error. The difference from the negative control is statistically significant: ${ }^{*} P<0.001$ (Tukey-Kramer test). The negative control $=$ PMNs incubated in Hanks' Balanced Salt Solution supplemented with $0.1 \%$ decomplemented fetal bovine serum and incubated with cytochalasin $\mathrm{B}$. This figure represents the results of at least three independent experiments. fMLP, $N$-formyl-methionyl-leucyl-phenylalanine.

capacity for PMNs to respond to phorbol myristate acetate [16].

Because $80 \mathrm{M}$ chitosan is chemotactic for PMNs, it must interact at the surface of PMNs to elicit a chemotactic response. The majority of chemotactic factors mediate their effect through G-protein-coupled receptors. To determine whether this applies to $80 \mathrm{M}$ chitosan, we assessed the effect of pertussis toxin on $80 \mathrm{M}$ chitosan-induced chemotaxis of PMN.
Pertussis-toxin inhibited PMN chemotaxis by $80 \%$, implicating a G-protein-coupled receptor. The mechanism through which $80 \mathrm{M}$ chitosan activates a G-protein-coupled receptor remains to be determined. It was previously found that conditioned media from canine PMNs stimulated with $>80 \%$ DDA chitosan particles promoted chemotaxis of neutrophils [14]. We provide evidence, using a specific $\mathrm{CPLA}_{2}-\alpha$ inhibitor, that phospholipid-derived mediators, possibly the chemotactic factors $\mathrm{LTB}_{4}$ and PAF, are involved in the direct chemotactic activity 
Figure 5

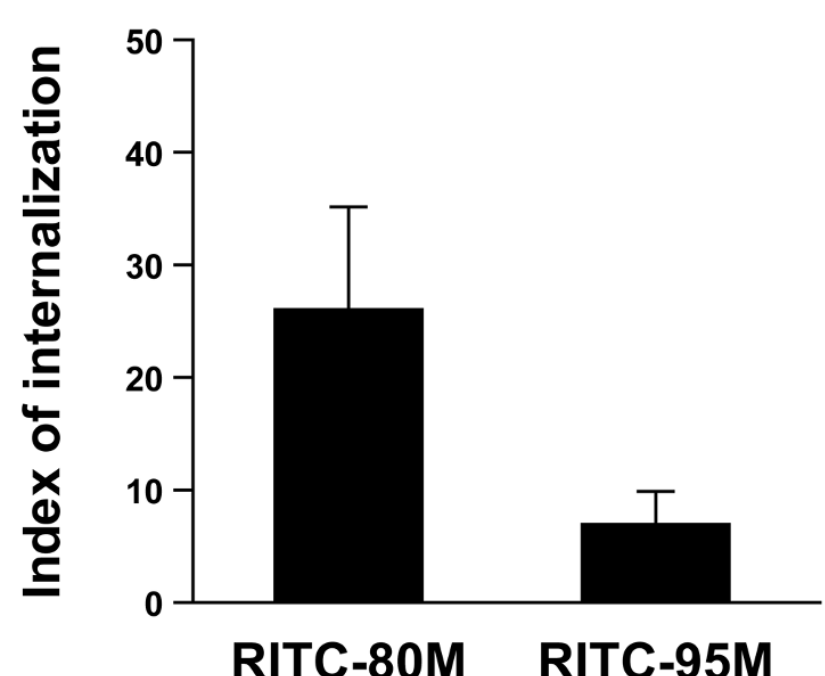

The internalization of chitosan by PMNs. Freshly isolated polymorphonuclear neutrophils (PMNs) were resuspended in RPMI 1640 supplemented with $0.1 \%$ decomplemented fetal bovine serum, pre-stained with $1 \mu \mathrm{g} / \mathrm{ml}$ calcein-AM for 30 minutes at $37^{\circ} \mathrm{C}$ and incubated with $100 \mu \mathrm{g} / 1 \times 10^{6}$ cells rhodamine B isothiocyanate (RITC)-zymosan for 1.5 hours (a positive control), $15 \mu \mathrm{g} / \mathrm{ml}$ RITC- $80 \%$ deacetylated ( $80 \mathrm{M})$ or RITC-95\% deacetylated $(95 \mathrm{M})$ chitosan for 3 hours at $37^{\circ} \mathrm{C}$. PMNs were then centrifuged and plated on a slide coated with $100 \%$ decomplemented autologous serum and visualized by live confocal microscopy. The index of internalization of chitosan by PMNs was calculated as the percentage of cells that internalized RITC-chitosan. Results are presented as mean \pm standard error. This figure represents the results of three independent experiments.

of human PMNs toward a pure and sterile $80 \mathrm{M}$ chitosan preparation. This is the first study to demonstrate that such lipid mediators contribute to half of the chemotactic activity of human PMNs toward chitosan. The $\mathrm{CPLA}_{2}-\alpha$ inhibitor pyrrolidine-1 inhibited the chemotaxis of PMNs by $50 \%$. Moreover, the inhibition of chemotaxis by $80 \%$ in the presence of pertussis toxin suggests that additional chemotactic agents acting through G-protein-coupled receptors participate in the chemotaxis of PMNs toward chitosan. Further investigation is required to characterize fully the molecular mechanisms that are involved in $80 \mathrm{M}$ chitosan-induced chemotaxis of human PMNs.

Having characterized the response of PMNs toward $80 \mathrm{M}$ chitosan, we conducted similar experiments with $95 \mathrm{M}$ chitosan because we had observed a distinct response of PMNs toward $95 \mathrm{M}$ chitosan in vivo. This is the first report on the effect of $95 \mathrm{M}$ chitosan on PMN effector functions. Chitosan $95 \mathrm{M}$ (95\% glucosamine, 5\% N-acetyl glucosamine) was unable to induce chemotactic activity, superoxide production, or the release of granule contents by PMNs. The lack of chemotactic activity of $95 \mathrm{M}$ chitosan toward PMNs was not due to an effect on the viability of PMNs (data not shown). The percentage DDA of chitosan is therefore a determining factor for
Figure 6

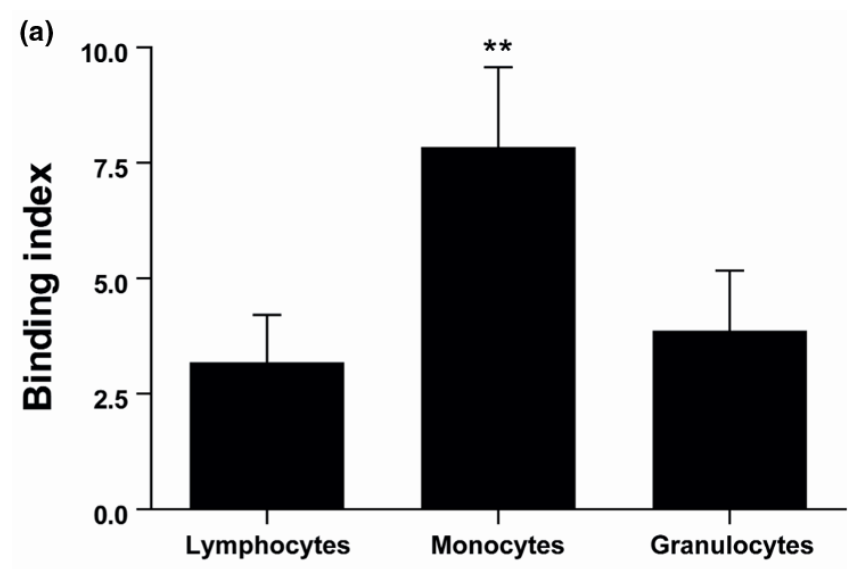

(b)
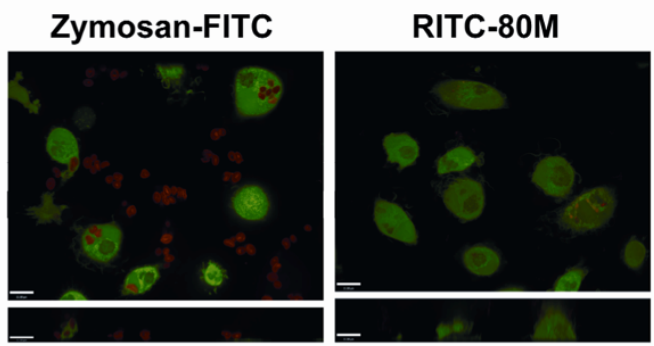

RITC-95M

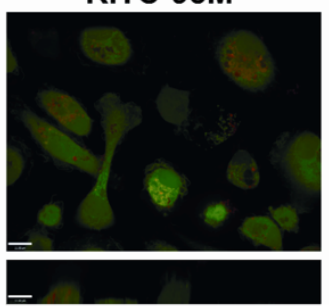

The interaction of chitosan with monocytes, granulocytes, and lymphocytes in whole blood. (a) Whole blood was incubated for 30 minutes with $5 \mu \mathrm{g} / \mathrm{ml}$ rhodamine B isothiocyanate (RITC)-80\%

deacetylated $(80 \mathrm{M})$ chitosan at $37^{\circ} \mathrm{C}$ for 30 minutes before analysis by flow cytometry. The binding index was calculated as fluorescence units of each leukocyte population incubated with RITC-80 M chitosan/fluorescence units of leukocyte population in the absence of RITC-chitosan. Results are presented as mean \pm standard error. $P$ values from Student's two-tailed unpaired $t$-test: ${ }^{\star \star} P<0.005$ versus autofluorescence for each leukocyte population. (b) Macrophages were seeded on glass slides $\left(2 \times 10^{6} \mathrm{cells} / \mathrm{ml}\right)$ in RPMl 1640 supplemented with $0.1 \%$ decomplemented fetal bovine serum, pre-stained with $1 \mu \mathrm{g} / \mathrm{ml}$ calcein AM for 30 minutes at $37^{\circ} \mathrm{C}$, and incubated with $100 \mu \mathrm{g} / 1 \times$ $10^{6}$ cells RITC-zymosan for 1.5 hours (a positive control), $15 \mu \mathrm{g} / \mathrm{ml}$ RITC-80 M, or RITC-95\% deacetylated (95 M) chitosan for 3 hours at $37^{\circ} \mathrm{C}$. Macrophages were then visualized live through a spinning disc confocal microscope with a $63 \times$ objective. The top panels are images taken in the $X-Y$ plane and the lower panels are images taken in the $X-Z$ plane. This figure represents the results of three independent experiments. 
the activation of PMNs by chitosan and potentially for the therapeutic use of chitosan. Our findings indicate that chitosans in the range from $80 \%$ to $95 \%$ DDA can elicit quite different biologic responses, and highlight the importance of defining the DDA level when conducting biologic assays. Some of the differential responses could be related to the very low solubility of $95 \%$ DDA chitosan at neutral pH [8]. In the light of these findings, it is of interest to investigate fully the effect of chitosan with other percentages of DDA on PMNs to determine whether there is a percentage DDA that induces maximal chemotactic activity in PMNs and consequently an optimal therapeutic effect.

Another parameter that may modify the response of PMNs to chitosan is the form in which the chitosan is used. Vandevord and coworkers [6] reported that a chitosan scaffold made with chitosan of $92 \%$ DDA is chemotactic for PMN in vivo. Because $92 \%$ DDA chitosan is structurally more similar to 95 $\mathrm{M}$ than to $80 \mathrm{M}$ chitosan, our data indicate that PMN migration to $92 \%$ DDA chitosan should be quite modest. The discrepancy between this previous observation and our findings could potentially be explained by the fact that the scaffold used in the in vivo study was prepared by coating polytetrafluoroethylene tubes with $92 \%$ DDA chitosan, and did not employ pure chitosan. It will be of therapeutic interest to determine how differently PMNs react to chitosans of the same percentage DDA of different structural forms - suspension versus scaffold.

It is generally accepted that PMN phagocytose chitosan, but no microscopy studies have been performed to demonstrate that PMNs indeed internalize chitosan. This is a relevant question because PMNs can respond to foreign material without necessarily internalizing it. We provide direct evidence that PMNs can internalize $80 \mathrm{M}$ chitosan without stimulating degranulation. Around $10 \%$ of PMNs internalized $80 \mathrm{M}$ chitosan, in the presence of $0.5 \%$ heat-inactivated serum. These observations are quite different to those in PMN and monosodium urate crystals, which have a poor capacity for internalization while strongly activating PMNs, probably because of an autocrine effect [25]. It is highly likely that lipid mediators are involved in this autocrine effect. In our internalization assay, PMNs readily internalized zymosan, a yeast cell wall preparation that activates neutrophils. Because both $95 \mathrm{M}$ and $80 \mathrm{M}$ were internalized without activating neutrophils, our data demonstrate that internalization of a polysaccharide biomaterial does not automatically trigger degranulation.

Recently, PMNs were reported to express the mannose receptor [26], a receptor that is implicated in the internalization of chitosan by macrophages $[27,28]$. We provide evidence that monocytes internalize $80 \mathrm{M}$ chitosan more readily than PMNs, suggesting that the molecular mechanisms involved in the internalization of $80 \mathrm{M}$ chitosan by PMNs differ from those of macrophages. This does not imply a less important role of PMNs in chitosan-based wound healing. PMNs usually out- number macrophages in certain phases of wound healing and can collectively synthesize large quantities of soluble mediators.

\section{Conclusions}

In summary, $80 \mathrm{M}$ chitosan is chemotactic for human PMNs but does not activate additional PMN effector functions such as degranulation and superoxide production. Because the beneficial therapeutic effects of $80 \mathrm{M}$ chitosan are preceded by the recruitment of a significant number of PMNs, this chitosan-induced PMN phenotype could be associated with promotion of repair. Our observations also indicate that the degree of deacetylation is an important factor to consider in the use of chitosan as an accelerator of repair because PMNs exhibit a differential capacity to migrate towards $80 \mathrm{M}$ and 95 $M$ chitosan.

\section{Competing interests}

The authors declare that they have no competing interests.

\section{Authors' contributions}

$P S$ and $H G$ made equal contributions to the experimental aspects of this study. They performed the majority of the experiments. SM and DR made important contributions to the conduct of certain experiments as well as the interpretation of the data. MF, PP, and $\mathrm{CH}$ contributed to the design of the experiments and the interpretation of the data. MF wrote the manuscript, and $\mathrm{PP}$ and $\mathrm{CH}$ revised it. All the experiments were performed and supervised in MF's laboratory. HEG contributed to the interpretation of the data.

\section{Acknowledgements}

This research was funded by a Discovery Advancement Program grant awarded by the Canadian Arthritis Network to CDH, MJGF, PEP, and HEG. PS is a recipient of a scholarship awarded by the Canadian Arthritis Network, MJGF received a salary award from The Arthritis Society, and $\mathrm{CDH}$ received a salary award from the Fonds de la Recherche Santé Québec. We should like to thank Dr Matthew Shive for insightful comments and critical reading of the manuscript.

\section{References}

1. Felson DT: An update on the pathogenesis and epidemiology of osteoarthritis. Radiol Clin North Am 2004, 42:1-9.

2. Hoemann CD, Hurtig M, Rossomacha E, Sun J, Chevrier A, Shive MS, Buschmann MD: Chitosan-glycerol phosphate/blood implants improve hyaline cartilage repair in ovine microfracture defects. J Bone Joint Surg Am 2005, 87:2671-2686.

3. Hoemann CD, Sun J, Mckee MD, Chevrier A, Rossomacha E, Rivard GE, Hurtig M, Buschmann MD: Chitosan-glycerol phosphate/blood implants elicit hyaline cartilage repair integrated with porous subchondral bone in microdrilled rabbit defects. Osteoarthritis Cartilage 2007, 15:78-89.

4. Shive MS, Hoemann CD, Restrepo A, Hurtig MB, Duval N, Ranger P, Stannish W, Buschmann MD: BST-CarGel: in situ chondroinduction for cartilage repair. Operative techniques in orthopaedics: articular cartilage surgery. Operative Techn Orthopaedics 2006, 16:271-278.

5. Onishi H, Machida Y: Biodegradation and distribution of watersoluble chitosan in mice. Biomaterials 1999, 20:175-182.

6. VandeVord PJ, Matthew HWT, DeSilva SP, Mayton L, Wu B, Wooley $\mathrm{PH}$ : Evaluation of the biocompatibility of a chitosan scaffold in mice. J Biomed Mater Res 2002, 59:585-590. 
7. Malette WG, Quigley HJ, Gaines RD, Johnson ND, Rainer WG: Chitosan - a new hemostatic. Ann Thorac Surg 1983, 36:55-58.

8. Varum KM, Holme HK, Izume M, Stokke BT, Smidsrod O: Determination of enzymatic hydrolysis specificity of partially $\mathrm{N}$ acetylated chitosans. Biochim Biophys Acta 1996, 1291:5-15.

9. Sashiwa H, Saimoto H, Shigemasa Y, Ogawa R, Tokura S: Lysozyme susceptibility of partially deacetylated chitin. Int J Biol Macromol 1990, 12:295-296.

10. Tomihata $\mathrm{K}$, Ikada $\mathrm{Y}:$ In vitro and in vivo degradation of films of chitin and its deacetylated derivatives. Biomaterials 1997, 18:567-575.

11. Chevrier A, Hoemann CD, Sun J, Buschmann MD: Chitosan-glycerol phosphate/blood implants increase cell recruitment, transient vascularization and subchondral bone remodeling in drilled cartilage defects. Osteoarthritis Cartilage 2007, 15:316-327.

12. Usami $Y$, Okamoto $Y$, Minami $S$, Matsuhashi $A$, Kumazawa $N H$, Tanioka S, Shigemasa Y: Migration of canine neutrophils to chitin and chitosan. J Vet Med Sci 1994, 56:1215-1216.

13. Usami $Y$, Okamoto $Y$, Minami $S$, Matsuhashi $A$, Kumazawa $N H$, Tanioka S, Shigemasa Y: Chitin and chitosan induce migration of bovine polymorphonuclear cells. J Vet Med Sci 1994, 56:761-762

14. Usami Y, Okamoto Y, Takayama T, Shigemasa Y, Minami S: Chitin and chitosan stimulate canine polymorphonuclear cells to release leukotriene B-4 and prostaglandin E-2. J Biomed Mater Res 1998, 42:517-522.

15. Ueno H, Yamada H, Tanaka I, Kaba N, Matsuura M, Okumura M, Kadosawa T, Fujinaga T: Accelerating effects of chitosan for healing at early phase of experimental open wound in dogs. Biomaterials 1999, 20:1407-1414.

16. Dou JL, Tan CY, Du YG, Bai XF, Wang KY, Ma XJ: Effects of chitooligosaccharides on rabbit neutrophils in vitro. Carbohyd Polym 2007, 69:209-213.

17. Ma O, Lavertu M, Sun J, Nguyen S, Buschmann MD, Winnik FM, Hoemann CD: Precise derivatization of structurally distinct chitosans with rhodamine B isothiocyanate. Carbohyd Polym 2008, 72:616-624.

18. Fernandes MJ, Rollet-Labelle E, Pare G, Marois S, Tremblay ML, Teillaud JL, Naccache PH: CD16b associates with high-density, detergent-resistant membranes in human neutrophils. Biochem J 2006, 393:351-359.

19. Gilbert C, Levasseur S, Desaulniers P, Dusseault AA, Thibault N, Bourgoin SG, Naccache PH: Chemotactic factor-induced recruitment and activation of $\mathrm{Tec}$ family kinases in human neutrophils. II. Effects of LFM-A13, a specific Btk inhibitor. J Immunol 2003, 170:5235-5243.

20. Bradley PP, Priebat DA, Christensen RD, Rothstein G: Measurement of cutaneous inflammation: estimation of neutrophil content with an enzyme marker. J Invest Dermatol 1982, 78:206-209.

21. Mocsai A, Ligeti E, Lowell CA, Berton G: Adhesion-dependent degranulation of neutrophils requires the Src family kinases Fgr and Hck. J Immunol 1999, 162:1120-1126.

22. Desmeules P, Dufour M, Fernandes MJ: A rapid flow cytometry assay for the assessment of calcium mobilization in human neutrophils in a small volume of lysed whole-blood. $J$ Immunol Methods 2009, 340:154-157.

23. Bauldry SA, Wooten RE: Leukotriene B4 and platelet activating factor production in permeabilized human neutrophils: role of cytosolic PLA2 in LTB4 and PAF generation. Biochim Biophys Acta 1996, 1303:63-73.

24. Iking-Konert C, Cseko C, Wagner C, Stegmaier S, Andrassy K, Hansch GM: Transdifferentiation of polymorphonuclear neutrophils: acquisition of CD83 and other functional characteristics of dendritic cells. J Mol Med 2001, 79:464-474.

25. Desaulniers P, Fernandes M, Gilbert C, Bourgoin SG, Naccache $\mathrm{PH}$ : Crystal-induced neutrophil activation. VII. Involvement of Syk in the responses to monosodium urate crystals. J Leukoc Biol 2001, 70:659-668.

26. Valera I, Vigo AG, Alonso S, Barbolla L, Crespo MS, Fernandez N: Peptidoglycan and mannose-based molecular patterns trigger the arachidonic acid cascade in human polymorphonuclear leukocytes. J Leukocyte Biol 2007, 81:925-933.
27. Mori T, Murakami M, Okumura M, Kadosawa T, Uede T, Fujinaga T: Mechanism of macrophage activation by chitin derivatives. Vet Med Sci 2005, 67:51-56.

28. Feng J, Zhao L, Yu Q: Receptor-mediated stimulatory effect of oligochitosan in macrophages. Biochem Biophys Res Commun 2004, 317:414-420. 